\title{
BMJ Open Association of occupational sitting with cardiovascular outcomes and cardiometabolic risk factors: a systematic review with a sex-sensitive/ gender-sensitive perspective
}

\author{
Kathrin Reichel (D) , ${ }^{1}$ Michaela Prigge, ${ }^{1}$ Ute Latza, ${ }^{1}$ Tobias Kurth (D) , ${ }^{2}$ \\ Eva-Maria Backé ${ }^{1}$
}

To cite: Reichel $\mathrm{K}$, Prigge $\mathrm{M}$ Latza U, et al. Association of occupational sitting with cardiovascular outcomes and cardiometabolic risk factors: a systematic review with a sex-sensitive/gender-sensitive perspective. BMJ Open 2022;12:e048017. doi:10.1136/ bmjopen-2020-048017

- Prepublication history and additional supplemental material for this paper are available online. To view these files, please visit the journal online (http://dx.doi.org/10.1136/ bmjopen-2020-048017)

Received 18 December 2020 Accepted 24 December 2021

Check for updates

(c) Author(s) (or their employer(s)) 2022. Re-use permitted under CC BY-NC. No commercial re-use. See rights and permissions. Published by BMJ.

${ }^{1}$ Work and Health, Federal Office for Health and Safety in the Workplace Berlin, Berlin, Germany

${ }^{2}$ Institute of Public Health, Charité Universitätsmedizin Berlin, Berlin, Germany

Correspondence to

Dr Eva-Maria Backé;

backe.eva-maria@baua.bund.de

\section{ABSTRACT}

Objectives Sedentary behaviour is a modifiable risk factor for cardiovascular health. Although long periods of sedentary behaviour take place at work, evidence of the relationship between such occupational sitting and cardiometabolic health risks remains limited. This systematic review aimed to update the evidence on the associations of occupational sitting with cardiovascular outcomes and cardiometabolic risk factors based on longitudinal studies.

Design Systematic review.

Setting Workplace.

Population Employees aged 18-65 years.

Primary and secondary outcomes Primary outcomes were cardiovascular diseases and cardiometabolic risk markers. The secondary outcome was all-cause mortality. Data sources Ten databases, including PubMed, Web of Science and CINAHL (search January 2018, updated February 2019).

Data extraction and synthesis Data were screened, extracted and appraised by three independent reviewers following the Preferred Reporting Items for Systematic Reviews and Meta-Analyses guidelines.

Results Studies were markedly heterogeneous in terms of measurement of occupational sitting, cardiometabolic risk factors and cardiovascular morbidity and mortality, so that standards were hardly identifiable and limiting the value of the evidence. The review included 27 high or acceptable quality publications. Of the eight high-quality publications from seven cohorts, three cohort studies found significant associations of occupational sitting with primary outcomes. Additionally, one study described an association with the secondary outcome. Another high-quality publication found an association between occupational sitting and ischaemic heart disease in a subgroup already at risk due to hypertension. For sex/ gender analysis, 11 of the 27 high and acceptable quality publications reported sex-stratified results. Five of these found sex differences.

Conclusions Evidence regarding the association of occupational sitting with cardiometabolic health risks was limited because of the lack of standardised measurements for occupational sitting. 0ccupational sitting combined
Strengths and limitations of this study

- This paper updated a previous review on occupational sitting and health risks based on longitudinal studies, with a special focus on cardiometabolic outcomes and sex/gender differences.

- Ten databases as well as references of the identified studies were included.

- Reliability of study selection, data extraction and risk-of-bias assessment was ensured by three independent reviewers.

- Findings were limited by the heterogeneity of measurements of occupational sitting and the wide variety of measured outcomes.

- Study heterogeneity precluded a meta-analysis.

with an overall sedentary lifestyle was associated with an elevated relative risk for several cardiometabolic outcomes. There is an urgent need for standardised measurements of occupational sitting to facilitate metaanalysis. Sex/gender aspects of this relationship require further investigation.

\section{INTRODUCTION}

Cardiovascular events are a leading cause of death worldwide. ${ }^{1}$ The contribution of sedentary behaviour to cardiovascular health risks is well-documented. ${ }^{2-4}$ Sedentary behaviour is defined as 'any waking behaviour characterised by energy expenditure $\leq 1.5$ metabolic equivalents, while in a sitting, reclining or lying posture. ${ }^{5}$ Occupational sitting is defined as sedentary behaviour in the workplace. Prolonged occupational sitting is potentially a relevant and modifiable risk factor of cardiovascular health, as $50 \%-60 \%$ of individuals' total sitting time is spent at work. ${ }^{6}$ However, the role of occupational sitting in the aetiology of cardiometabolic outcomes remains unclear. 
To date, only one systematic review ${ }^{7}$ has summarised studies of the association of occupational sitting with body mass index (BMI), cardiovascular disease (CVD), diabetes mellitus, cancer and mortality until 2009, but found heterogeneous results. There has been no more recent update of the literature on the association between occupational sitting (as a work-related exposure) and cardiometabolic outcomes based on longitudinal studies. Yet, this is needed as a basis for recommendations by stakeholders in the field of occupational safety and health.

The onset and incidence of CVD differs between women and men $^{8}$ due to sex-related biological differences, as well as sociocultural differences that impact the risk for CVD. Additionally, there are sex differences in the health benefits derived from physical activity, ${ }^{9}$ and there are gender differences in terms of physical inactivity and sedentary behaviour in different domains. ${ }^{6} 1011$ There is a strong horizontal and vertical segregation in the labour market between men and women with similar trends across European countries. ${ }^{12}$ For sedentary work, horizontal segregation results in different sitting demands, for example, women mostly sit during computer work, while men often sit while driving. A gender-sensitive, comprehensive data analysis requires description of the gender bias of the studies included in such an analysis, and careful interpretation of sex-stratified analyses based on a general framework ${ }^{13-15}$ with a focus on the workplace. ${ }^{16}$ As shown above, sex-based biological factors and gendered social factors are interrelated in this context. Recognising the entanglement of the terms 'sex' and 'gender', hereinafter we use the term 'sex/gender' recommended by the Cochrane Sex/Gender Methods Group. ${ }^{15}$

Thus, the aim of the present systematic review was to update the evidence ${ }^{7}$ regarding the association between occupational sitting and cardiometabolic outcomes, based on longitudinal studies with a sex-sensitive/gendersensitive perspective.

\section{METHODS}

The research protocol for this systematic review, including the search strategy, was registered in advance (PROSPERO registration: CRD42018079219),${ }^{17}$ and inclusion criteria were defined following the patient, intervention (exposure), comparison, outcome and setting (PICO) model. The review is reported according to the Preferred Reporting Items for Systematic Reviews and Meta-Analyses (PRISMA) statement. ${ }^{18}$

\section{Search strategy}

The list of search terms was based on the previous review on this topic. ${ }^{7}$ The search string included terms of sedentary behaviour, as an exposure, in combination with an approved search string for work-related inquiries ${ }^{19}$ (figure 1). We intentionally used a broad and comprehensive search string, with no restrictions on diseases or cardiometabolic outcomes. The search strategy was developed by a senior researcher and the project leader (UL,
General approach for search string:

Setting work (Mattioli et al. 2010) AND exposure sitting: Search in title/abstract and keywords

- Limitations: 2009-2017

- Restricted to European languages

- Restricted to peer reviewed journals, articles

\section{Exemplary Search String for data base searches for PubMed (including MEDLINE)}

1. Work context (Mattioli et al 2010)

occupational diseases $[M H]$ OR occupational exposure $[M H]$ OR occupational exposure* [TW] OR "OCcupational health" OR "occupational medicine" $O R$ work-related $O R$ working environment [TW] $O R$ at work [TW] OR work environment [TW] OR occupations [MH] OR work [MH] OR workplace ${ }^{\star}[T W] O R$ workload $O R$ occupation* $O R$ worke* $O R$ work place $[T W]$ OR work site* [TW] OR job* [TW] OR occupational groups $[M H] O R$ employment $O R$ worksite* $O R$ industry $O R$ employee

2. AND sitting at the workplace

sedentar ${ }^{*}[T W]$ OR sitting [TW] OR seated [TW]

\section{Complete search string: 22.01 .2018 : result 2805 hits}

\section{(search in all fields)}

(( (occupational diseases[MH] OR occupational exposure[MH] OR occupational exposure*[TW] OR "occupational health" $O R$ "occupational medicine" $O R$ work-related $O R$ working environment[TW] $O R$ at work [TW] $O R$ work environment[TW] OR occupations $[M H] O R$ work $[M H] O R$ workplace $[T W] O R$ workload $O R$ occupation* $O R$ worke* $O R$ work place* [TW] OR work site* $[T W]$ OR job* [TW] OR occupational groups $[M H] O R$ employment $O R$ employee $O R$ worksite* $O R$ industry)) AND (sedentar*[TW] OR sitting[TW] OR seated[TW])) AND ("2009/03/01"[Date - Publication]: "2017/12/31"[Date - Publication])

Filters activated: Publication date from 2009/03/01 to 2017/12/31

Figure 1 Representative search strategy, showing the data search strategy used in PubMed.

E-MB), in consultation with an academic librarian, and was piloted by one researcher (KR) and calibrated by another senior medical researcher (FL).

Relevant publications were identified in January 2018 by literature searches in 10 databases, namely, the Cochrane Central Register of Controlled Trials, CINAHL, Embase, LIVIVO, OSH UPDATE, Physiotherapy Evidence Database, PsychINFO, PubMed, SPORTDiscus and Web of Science, and included reports published from 2009 to 2017. After systematic evaluation of the data sources of the included publications, the search was updated until February 2019 in four databases (PsychINFO, Embase, PubMed and Web of Science) in which all of the relevant publications were first identified. Reference of included publications were cross-checked for potential additional records. To manage data screening, abstrac and citation tracking, the reference management software package EndNote X9 was used.

In this review, we distinguished between the terms 'publications' and 'studies'. We found multiple fulltext publications that analysed different outcomes from the same longitudinal cohort or case-control study, for example, the Whitehall II cohort study, ${ }^{20-22}$ Danish Working Environment cohort study (DWECS) ${ }^{23-25}$ and the INTERHEART case-control study (a large international case-control study in different countries). ${ }^{26} 27$

\section{Inclusion criteria}

Based on the PICO scheme for structuring quantitative searches, the inclusion criteria were as follows: 
- A study population of gainfully employed adults, with a working age assumed to be 18-65 years.

- Exposure to sedentary behaviour in the workplace.

- Comparison of working populations with various amounts of sedentary time, or different physical job demands in the workplace.

- Outcomes including risk of CVDs, metabolic diseases, cardiometabolic mortality, or changes in cardiometabolic risk markers.

- A workplace setting.

- Cohort, case-control or interventional study designs.

Original, full-text, peer-reviewed articles published between April 2009 and February 2019 were included. For other reports, for example, congress presentations, abstracts or short reports, authors were requested access to original publications and study reports by email. Articles were restricted to peer-reviewed publications, with the intention of including high-quality studies, and were restricted to publications in all 24 European languages ${ }^{28}$ required by the funding bodies due to this study.

Following the PRISMA statement, ${ }^{18}$ three reviewers (KR, E-MB, MP) screened the records independently and in tandem by title and abstract and identified eligible publications. Subsequently, these reviewers identified eligible publications by screening the fulltext reports, using standardised forms. In the case of disagreement, consensus was sought by discussion, and if necessary, a deciding opinion was obtained from another reviewer (UL).

Data from included publications were extracted in duplicate. To ensure high accuracy and completeness, data extraction was checked and confirmed by three evaluators (KR, E-MB, MP).

Interventional studies were included but given the considerable differences in terms of study length, the measurement of exposure and subclinical outcomes (eg, lipids, glucose levels), they were evaluated separately. Studies with experimental designs were excluded. Additionally, studies investigating adolescents, subjects over 65 years of age or adults not gainfully employed exclusively were excluded.

\section{Risk-of-bias assessment and methodological assessment}

The methodology of publications, including potential risk-of-bias, was assessed using the methodology checklists for cohort and case-control studies of the SIGN network. ${ }^{29}$ Subquestions related to specific exposures, outcomes (including selective reporting) and confounders were added. The overall rating of each study was determined by consensus between the evaluators (KR, E-MB, MP) and, if necessary, by an independent reviewer (UL). The following overall ratings were assigned: 'high quality' $(++)$, indicating a study with low risk for confounding and bias; 'acceptable quality' $(+)$ and 'unacceptable/low quality' (0). Publications with low quality were excluded from the narrative summary of findings. ${ }^{30-32}$

\section{Sex-sensitive/gender-sensitive perspective}

Findings of sex-stratified analyses and studies with only male or female populations were also analysed and summarised.

\section{Measures of effect}

Depending on the statistical methods employed in the included articles, principal risk estimates were proportional hazards, from Cox and Poisson regression models in cohort studies and ORs in case-control studies, reported with $95 \%$ CIs and $p$ values. In cohort studies examining BMI, the BMI change $(\Delta)$ or obesity, $\beta$ risk estimate or risk estimate for $\Delta$ (regression analysis) were reported.

\section{Data synthesis}

Included articles were summarised by study designs. Publications were grouped according to the method for analysing exposure to occupational sitting. After data extraction and methodological assessment of included publications, the potential for a meta-analysis and pooling data was considered. We also performed subgroup analyses by cardiovascular risk groups (eg, participants with hypertension, overweight, metabolic syndrome (MS) or different BMI levels) in the included publications.

\section{Patient and public involvement statement}

We did not directly include patient and public involvement in this study as it is a systematic review with literaturebased data.

\section{RESULTS}

Study selection and systematic review process

Figure 2 presents the identified articles and selected publications according to the PRISMA statement. ${ }^{18}$ The 30 identified publications included 24 studies (19 cohort and 5 case-control studies). Six of these 30 publications reported more than one outcome, 5 included subgroup analyses on subjects already at risk of CVD and 12 included sex-stratified analyses.

\section{Study setting and study characteristics}

Characteristics of the 30 included publications are shown in tables 1 and 2. There were 17 studies from Europe, 5 from North America, 7 from Asia and 1 publication with data obtained from 52 countries. The number of participants ranged from $228^{33}$ to $134596 .{ }^{34}$ The mean follow-up duration varied between $3.3^{35}$ and 19.2 years. ${ }^{36}$ Occupational groups included healthcare, white collar and mixed cohorts of white-collar and blue-collar workers, agriculturists, office workers and general working population. Results were to be analysed according to the outcomes of the included studies, as per the study protocol. ${ }^{17}$ 


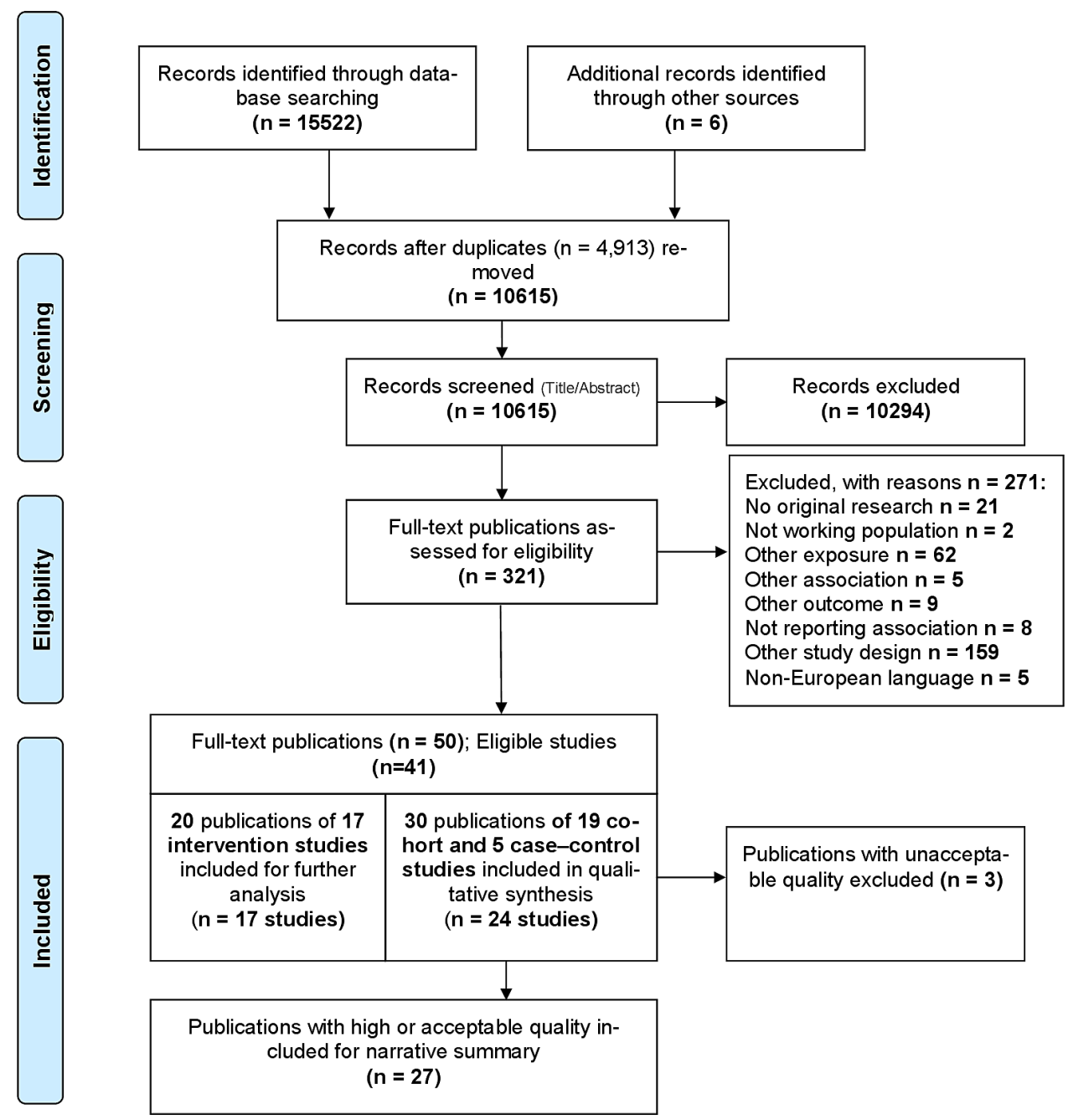

Figure 2 Preferred Reporting Items for Systematic Reviews and Meta-Analyses flow chart of the systematic review process.

\section{Risk of bias}

Methodological quality and risk-of-bias rating indicated acceptable to high quality of the publications (tables 1 and 2). The limitations were predominantly related to self-reported measurement of exposure. No cohort or case-control study used device-measured data (tables 1 and 2).

The relevant confounders in this context were age, sex, leisure time physical activity (LTPA), health behaviour (alcohol, smoking, diet) and socioeconomic status (SES). These main potential confounders were considered in nearly all studies, although LTPA was not considered in four 26273237 and SES was not considered in six publications. ${ }^{38-43}$

\section{Heterogeneous assessments of exposure to occupational sitting}

Among the included studies, exposure to occupational sitting was assessed in three ways: on a continuous scale or in categories as self-estimated amounts of time sitting at work, or as self-reported sedentary, low occupational physical activity (OPA), or by a job exposure matrix, as illustrated in table 3 .
In 11 of 30 publications, occupational sitting was analysed on a continuous scale or in categories as self-estimated amounts of time sitting at work, mostly based on a single question, "How many hours per week do you spent sitting at work?" Categories varied, for example, from self-report of occupational sitting time using a 5-point Likert scale, representing 'never' to 'always', ${ }^{43}$ to using different cutoff values, for example, $<24$ hours per week vs $\geq 24$ hours per week, ${ }^{23}$ or short ( $<1$ hour per day), middle 1-3 hours per day and longer $\geq 3$ hours per day. ${ }^{40}$

In 18 publications, exposure to occupational sitting was assessed as the least demanding OPA as compared with other categories, and heavy manual work as high OPA. Some studies used the 'Saltin-Grimby Physical Activity Level Scale'. ${ }^{44}$ Self-rated OPA was categorised into four classes, with different adaptations, labelling the categories in terms of walking and lifting, with no standardised norm discernible among the included studies. Three studies assessed exposure to occupational sitting using different types of job exposure matrices.

Six studies used standardised questionnaires, such as the Baecke Physical Activity Questionnaire. All standardised questionnaires contained either self-reported 


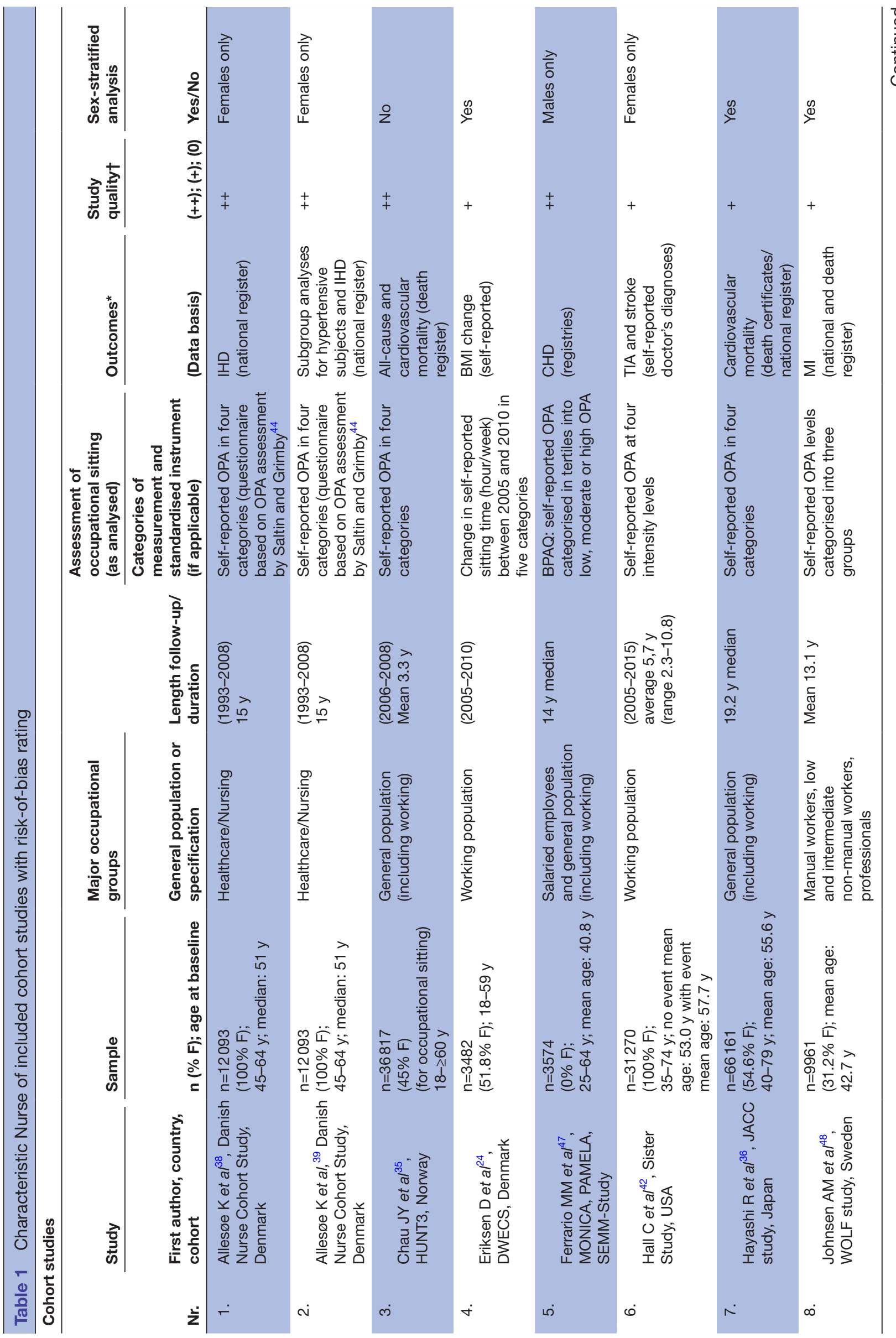




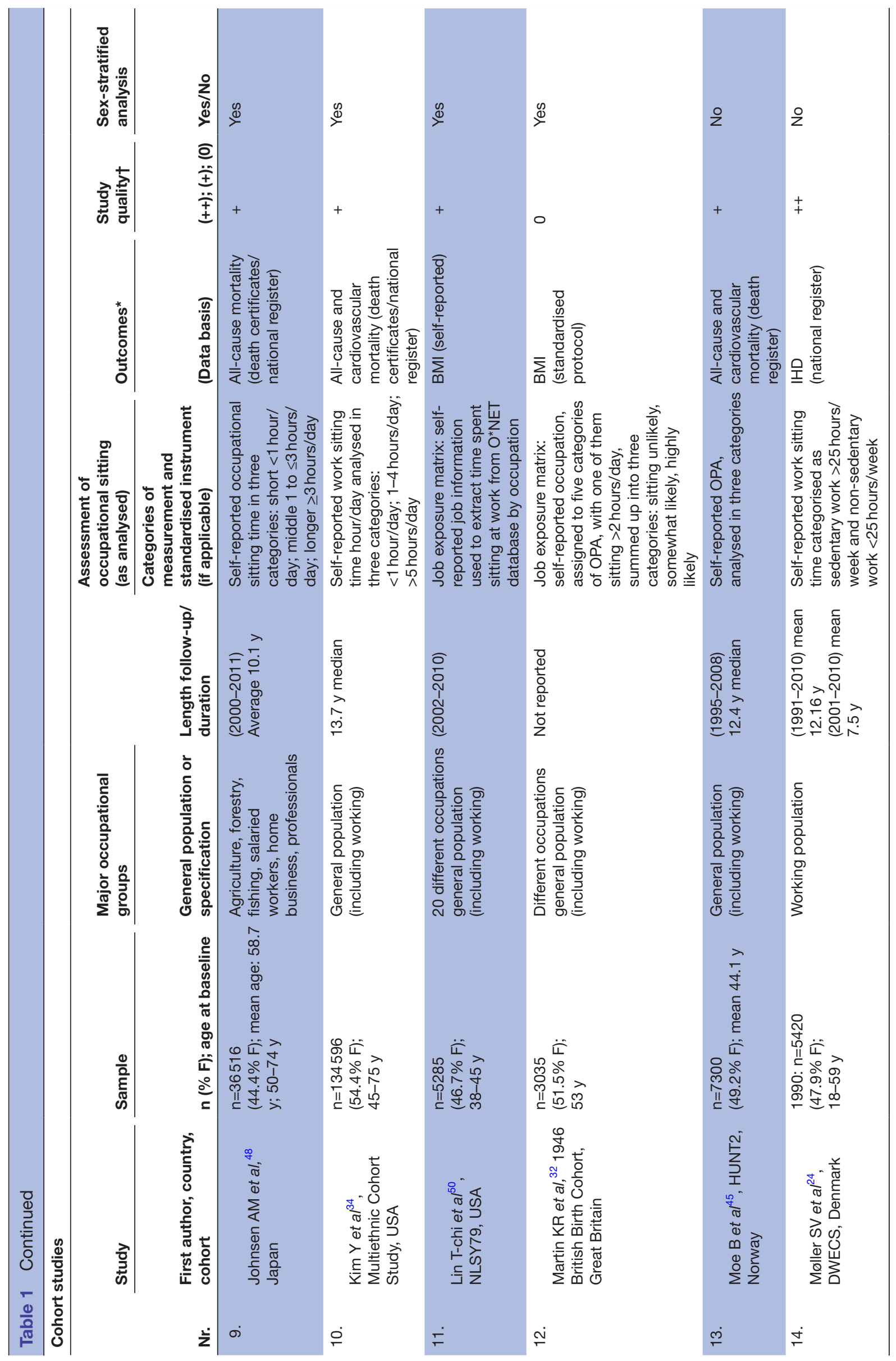

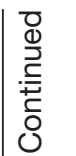




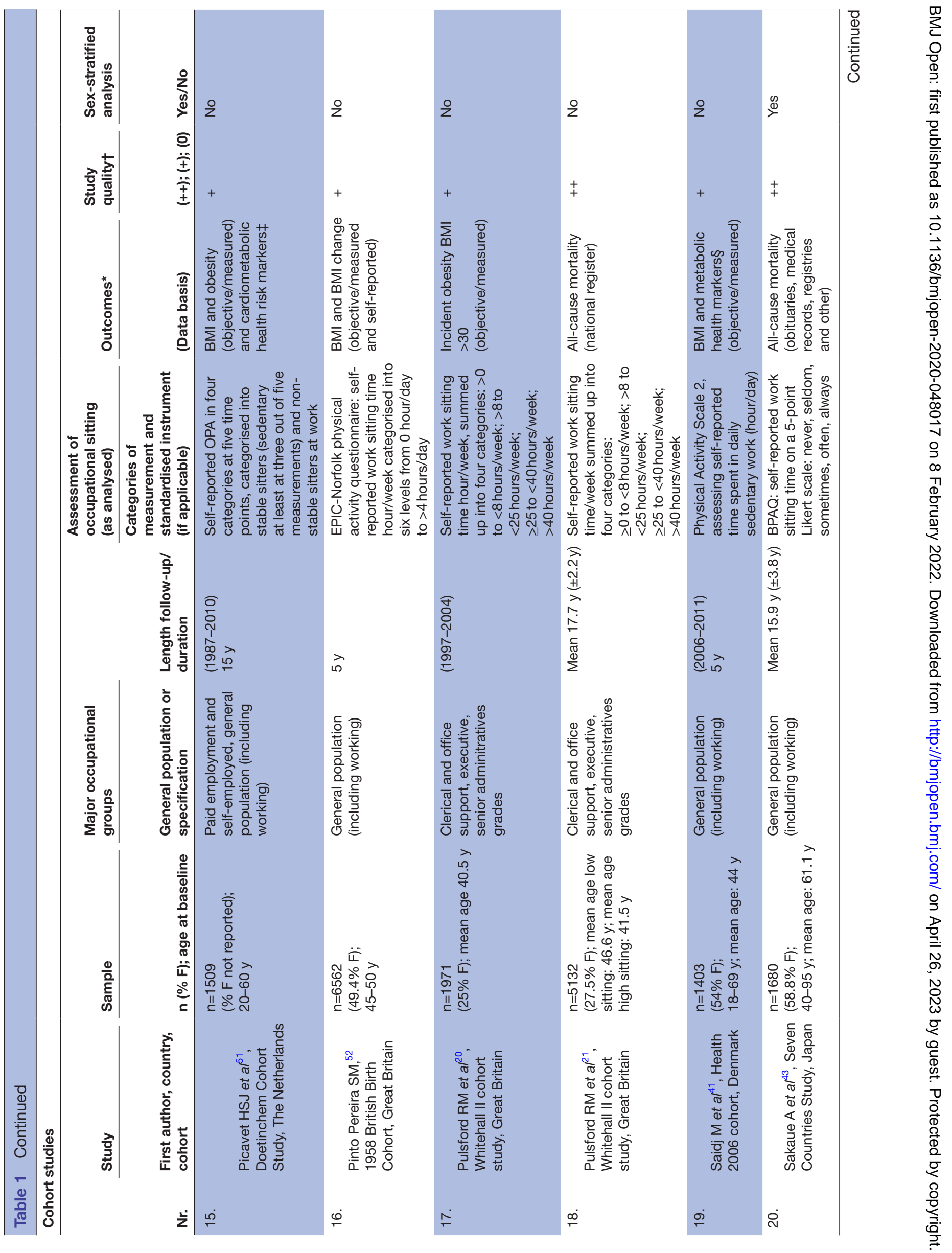




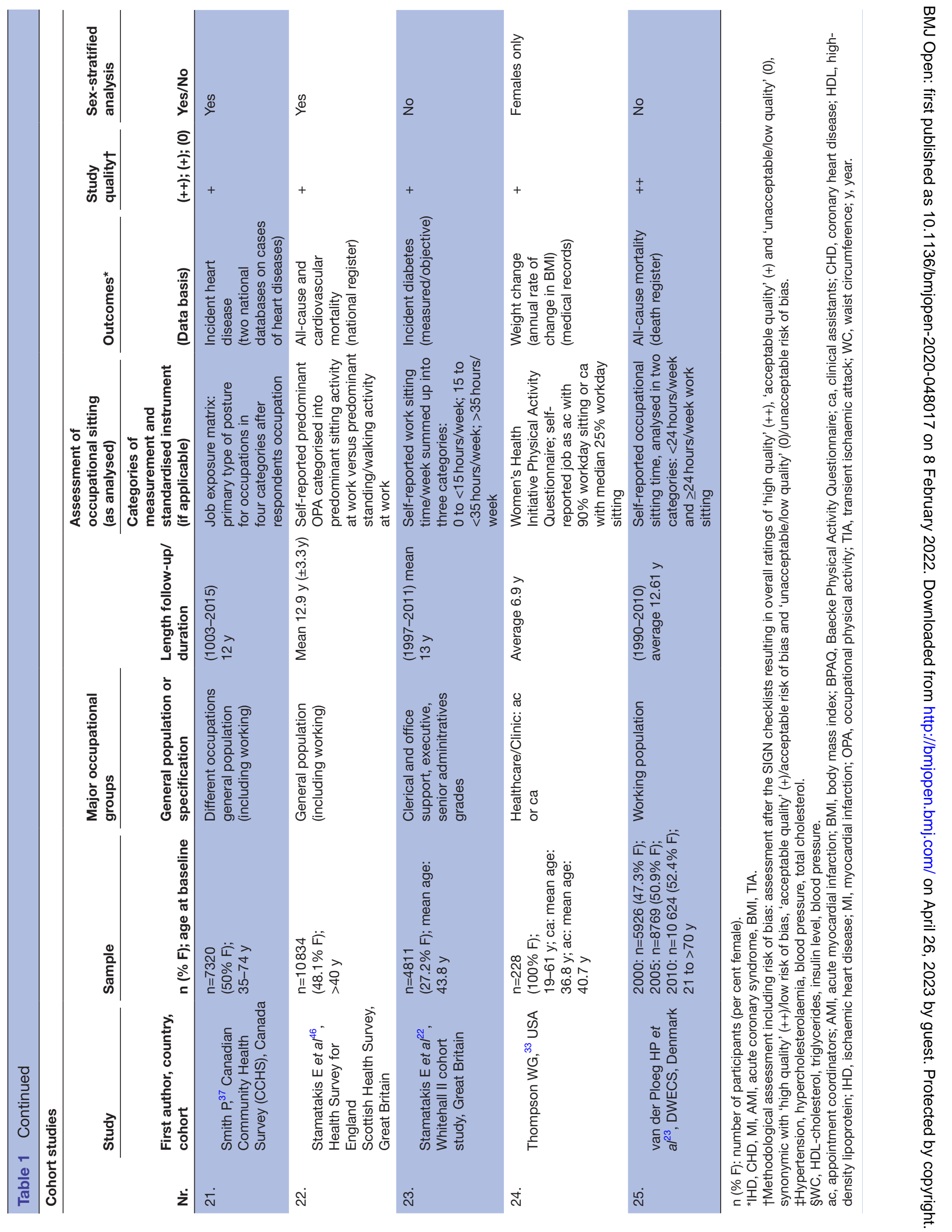




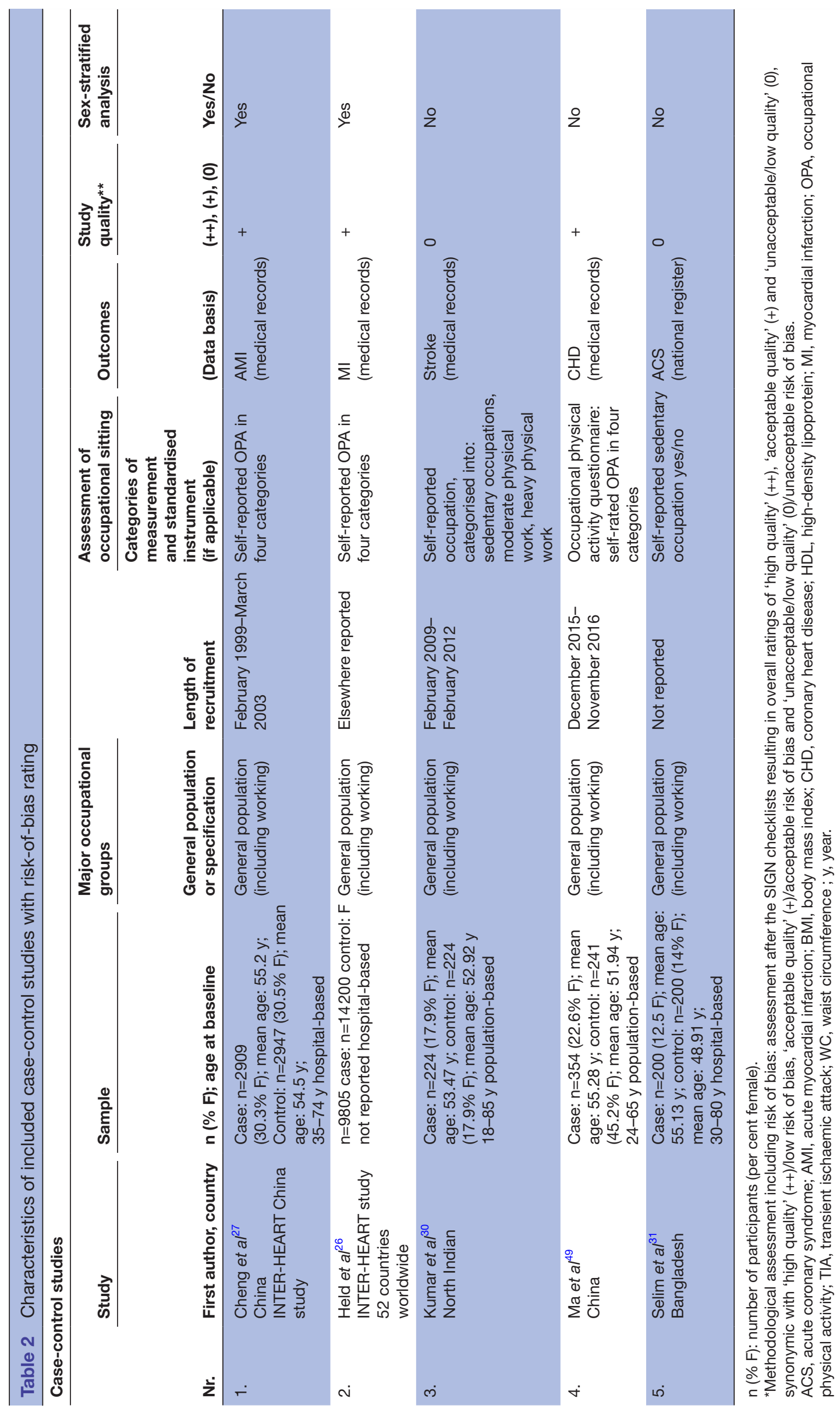


Table 3 Qualitative summary of findings by measurement of exposure and outcomes

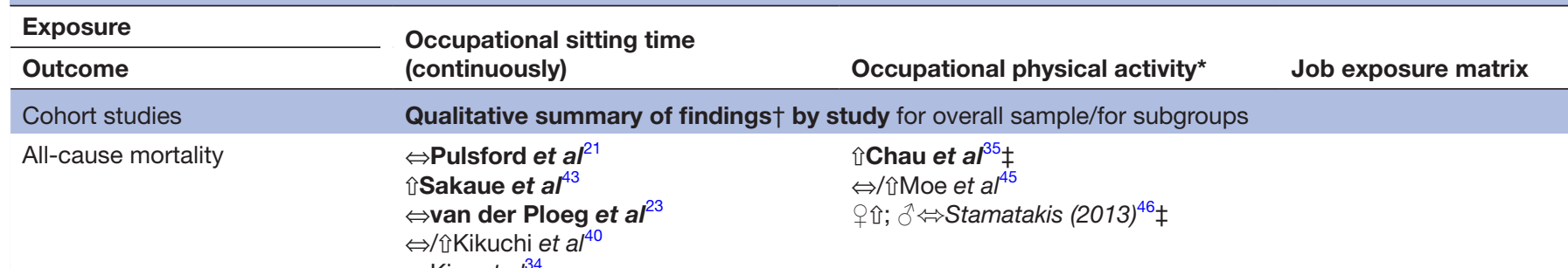

\begin{tabular}{|c|c|c|c|}
\hline Cardiovascular mortality & $\Leftrightarrow$ Kim et $\left.a\right|^{34}$ & 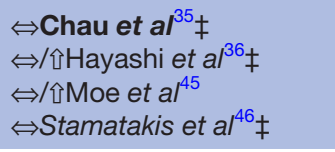 & \\
\hline
\end{tabular}

\begin{tabular}{|c|c|c|c|}
\hline $\begin{array}{l}\text { Cardiovascular morbidity (CHD } \\
\text { and stroke) }\end{array}$ & & 仓Ferrario et $a{ }^{47}$ & \\
\hline $\begin{array}{l}\text { Transient ischaemic attack and } \\
\text { stroke }\end{array}$ & & $\Leftrightarrow$ Hall et al ${ }^{42}$ & \\
\hline $\begin{array}{l}\text { Other cardiometabolic risk } \\
\text { markers }^{\star \star}\end{array}$ & $\begin{array}{l}\Leftrightarrow \text { Picavet et a }{ }^{51}(H T, H C L) \\
\Leftrightarrow \text { Stamatakis et } a l^{22}(D M)\end{array}$ & $\Leftrightarrow /(\mathrm{WC}) \Uparrow$ Saidj et al ${ }^{41}$ & \\
\hline \multicolumn{4}{|l|}{ Case-control studies } \\
\hline \multicolumn{2}{|l|}{ Cardiovascular heart diseasesף } & $\begin{array}{l}\Leftrightarrow / \delta \uparrow \text { Cheng et }\left.a\right|^{27} \ddagger(A M I) \\
\text { iHeld et } a l^{26} \ddagger(M I) \\
\uparrow M a \text { et } a l^{49}(C H D)\end{array}$ & \\
\hline
\end{tabular}

OFemale; ômale.

${ }^{*}$ Risk-of-bias rating is indicated by lettering: bold for low risk-of-bias (high quality) rated studies, cursive/italic for acceptable risk-of-bias rating. $\dagger$ †xplanation for qualitative summary of findings: îstatistical significant positive associations of exposure and outcome reported. $\Leftrightarrow$ No statistical significant association reported.

łIndicated if highest category of occupational sitting or sedentary/low occupational physical activity is reference group for statistical analysis. शIIHD, CHD, MI, AMI

**WC, HDL-cholesterol, triglycerides, insulin level, blood pressure, $\mathrm{HT}, \mathrm{HCL}, \mathrm{DM}$.

ACS, acute coronary syndrome; AMI, acute myocardial infarction; BMI, body mass index; CHD, coronary heart disease; DM, diabetes mellitus; HCL, hypercholesterolaemia; HDL, high-density lipoprotein; HF, heart failure; HT, hypertension; IHD, ischaemic heart disease; MI, myocardial infarction; WC, waist circumference.

occupational sitting time, OPA or job information. Other studies used adapted or self-constructed questionnaires to measure occupational sitting.

(Online supplemental table 1 demonstrates the heterogeneous nature of exposure assessment, exemplified for studies examining the association between occupational sitting and all-cause mortality. Online supplemental tables 2-7 describe details of the types of self-reported questionnaires or 'job exposure matrices' that were used.)

\section{Study heterogeneity}

Studies showed marked heterogeneity. Sources of heterogeneity included different populations (eg, only male subjects or only female subjects, or major occupational groups), exposure measurements, reference categories and outcomes. The varied reference categories are illustrated in online supplemental table 1 for studies examining the association between occupational sitting and all-cause mortality. Reference categories of those studies quantifying occupational sitting time ranged from $<8$ hours per week of occupational sitting ${ }^{21}$ to $<24$ hours per week of occupational sitting. ${ }^{23}$ Due to study heterogeneity, meta-analysis was impossible. Consequently, we have included a narrative summary of findings, from which publications with unacceptable quality were excluded. ${ }^{30-32}$

\section{Association between occupational sitting and all-cause mortality}

Eight cohort studies examined the association between occupational sitting and all-cause mortality, including four high-quality ${ }^{21} 233543$ and four acceptable-quality publications $^{34404546}$ (online supplemental table 2). Among the former, Chau et $a \ell^{35}$ reported that participants who selfrated their jobs as requiring 'much walking and lifting' had a $35 \%$ lower risk of all-cause mortality than those who reported sitting most of the time. Sakaue et $a l^{43}$ reported that occupational sitting time was significantly associated 
with all-cause mortality in male subjects only, Pulsford $e t$ $a l^{21}$ and van der Ploeg $e t a l^{23}$ reported no difference in allcause mortality between participants sitting for different durations.

Among the four acceptable-quality studies, Kim et $a l^{4}$ found no association between occupational sitting and all-cause mortality, for either sex. Kikuchi $e t a t^{t 0}$ reported a borderline association of longer occupational sitting time with all-cause mortality in a subsample of male primary industry workers. Moe $e t a t^{45}$ found a significant association in a subgroup with MS, but not in the overall sample. Stamatakis $e t a t^{6}$ found that a standing/walking occupation among women posed a lower risk of all-cause mortality than a sitting occupation.

\section{Association between occupational sitting and cardiovascular mortality}

Five cohort studies examined the association between occupational sitting and cardiovascular mortality, including one high-quality ${ }^{35}$ and four acceptable-quality studies $^{34} 364546$ (online supplemental table 3). The highquality study found no difference in risk for participants with mostly sitting jobs compared to jobs with higher OPA. ${ }^{35}$ The remaining prospective cohort studies reported no associations for overall samples. ${ }^{343645} 46$ For a subsample of overweight individuals, Hayashi et $a l^{36}$ reported a significantly higher risk for participants with MS, as well as Moe et $a l^{45}$

\section{Association between occupational sitting and cardiovascular heart diseases}

Eight publications examined the association between occupational sitting and CVDs. Of these, three cohort studies $^{25} 383947$ had high and two had acceptable quality, ${ }^{37} 48$ while three case-control studies had acceptable quality ${ }^{26279}$ (online supplemental table 4).

Ischaemic heart disease (IHD), myocardial infarction (MI), coronary heart disease (CHD) as well as acute coronary syndrome were considered as outcomes. Among the three high-quality publications, Møller et $a 2^{25}$ showed no difference in IHD risk between sedentary and nonsedentary employees. Allesøe $e t a l^{38}$ showed no significant association of sedentary work with increased IHD risk in female nurses, but found an association in those who were hypertensive. ${ }^{39}$ Ferrario et $a l^{47}$ compared low and moderate OPA in males, and found a significant CHD risk starting 3-5 years after baseline. Both acceptable-quality cohort studies found no association. ${ }^{374}$ Among the casecontrol studies, Ma et at $t^{49}$ showed significant association between sedentary occupations and CHD risk, particularly for participants working prolonged hours. They observed a significant linear relationship between OPA and CHD: the lesser the OPA during working, the higher the incidence of CHD. Held $e t a l^{26}$ showed a significantly reduced MI risk for occupations predominantly involving walking at one level and walking uphill and lifting objects, compared with sedentary work. Cheng $e t a l^{27}$ showed that associations between OPA levels and acute MI (AMI) risk were not linear, and that walking, compared with mostly sitting, at work reduced AMI risk in women.

Association between occupational sitting and cardiovascular morbidity and stroke

Two studies (one high-quality ${ }^{47}$ and one acceptablequality $^{42}$ ) reported an association between occupational sitting and cardiovascular morbidity and stroke (online supplemental table 5). Ferrario et $a t^{47}$ reported that low OPA posed a higher risk for CHD and stroke than intermediate OPA in working men. Hall et $a t^{42}$ reported no associations between sitting/standing OPA and transient ischaemic attack (TIA), compared with mostly sitting.

\section{Association of occupational sitting with BMI and obesity}

Seven acceptable-quality cohort studies examined the association between occupational sitting and BMI, BMI change or obesity ${ }^{2024334150-52}$ (online supplemental table 6). One study found a significant trend for female but not for male subjects. ${ }^{24}$ Lin et a ${ }^{50}$ showed that more sitting was significantly associated with higher BMI in men but not in women. Thompson $e t a l^{33}$ found a borderline association of sitting with BMI increase in women. Four studies found no association of occupational sitting with BMI and obesity, ${ }^{51}$ BMI or BMI change, ${ }^{52}$ incident obesity ${ }^{20}$ or 5 -year BMI change. ${ }^{41}$

\section{Association of occupational sitting with other cardiometabolic} risks and risk markers

Three acceptable-quality publications of cohort studies examined the association between occupational sitting and different cardiometabolic risk markers ${ }^{22} 4151$ (online supplemental table 7). Stamatakis $e t a l^{22}$ found no significant association of occupational sitting with incident diabetes. Picavet et a ${ }^{\tilde{1}}$ reported that stable sitting at work was not associated with hypertension or hypercholesterolaemia. Saidj $e t a l^{11}$ showed association of increased sitting time with waist circumference, but not other cardiometabolic health markers.

\section{Findings regarding sex/gender}

All 27 high-quality and acceptable-quality publications reported results adjusted for sex, and 12 provided a sexstratified analysis.

\section{Findings of publications with sex-stratified analysis}

Twelve of 27 publications reported sex-stratified findings concerning all-cause and cardiovascular mortality, ${ }^{3436404346}$ CHDs, ${ }^{26273748}$ BMI change ${ }^{24}$ and BMI ${ }^{3250}$ (online supplemental tables 2-7). One low-quality publication ${ }^{32}$ was excluded from the narrative summary.

Of the 11 included sex-stratified publications, associations of occupational sitting with mortality ${ }^{43} 46$ and $\mathrm{AMI}^{27}$ in females were found in three studies, and with BMI alone in males ${ }^{50}$ with BMI change in females ${ }^{24}$ and with MI in both sexes ${ }^{26}$ in one study each.

Six publications found no differences in risks between women and men, ${ }^{26} 3436373948$ five found no associations and one found an association in both sexes. 


\section{Female-only publications}

In four female-only publications, one study reported no association with IHD, ${ }^{38}$ except for hypertensives. ${ }^{39}$ One study ${ }^{42}$ reported no difference in risk for TIA and stroke among females with low-level OPA (mostly sitting, compared with those who were sitting and standing or mostly standing). One study ${ }^{33}$ found a borderline significant association of predominantly sitting, as compared with standing/walking at work, with BMI increase in women.

\section{Male-only publications}

Ferrario $e t a l^{47}$ showed associations of low OPA with CHD and cardiovascular morbidity (CHD and stroke) in men.

\section{Additional stratified analyses}

In 6 of the 27 publications, additional analyses examined the combined effect of different levels of OPA (low/ moderate/high) and LTPA or other physical activity levels with cardiometabolic outcomes. ${ }^{22} 3638414748$ Four of these found an association, and two did not. The combined effects of different levels of OPA and LTPA showed complex associations, rather than a dose-response relationship with cardiometabolic health risk for occupational sitting.

\section{Summary of risk estimates}

With regard to the multiple cardiovascular outcomes investigated across studies, most publications examined all-cause or cardiovascular mortality $(n=10)$, CHDs $(\mathrm{n}=5)$ or BMI $(\mathrm{n}=8)$ as an outcome. Of the 27 publications included in the narrative synthesis, 9 publications ( $\mathrm{n}=4$ with high-quality, $\mathrm{n}=5$ with acceptable-quality) showed that occupational sitting increased cardiometabolic risk (table 3). No study found a lower risk of the defined outcomes with sitting. One high-quality study reported a risk estimate of 1.28 for occupational sitting for cardiometabolic outcomes. ${ }^{43}$ Another high-quality cohort study ${ }^{35}$ reported a $35 \%$ lower risk for employees who were walking and lifting weights compared with those who were sitting. High-quality studies comparing groups with higher OPA to lower levels (sitting) reported risk estimates of $1.22^{38}$ to 1.61 . $^{47}$

Additionally, five subgroup analyses showed a noticeable effect on at-risk persons with hypertension, ${ }^{39} \mathrm{MS},{ }^{45}$ overweight $^{36}$ or working prolonged hours. ${ }^{49}$

\section{DISCUSSION}

In this systematic review of studies considering the effect of occupational sitting on cardiometabolic outcomes, we identified eight high-quality publications that presented results from seven cohort studies. Three cohort studies $^{38} 394347$ found significant associations of occupational sitting with the primary outcomes. The study by Chau et $a l^{85}$ reported no association with the primary outcome CVD mortality but with the secondary outcome all-cause mortality. In a subanalysis of the study by Allesøe et $a l^{39}$ occupational sitting was associated with IHD in the subgroup already at risk due to hypertension. ${ }^{39}$ The strength of the association in the included high-quality studies was weak to moderate.

The results based on the high-quality studies are similar to the results when additional 19 publications with at least acceptable quality are considered. Out of all 27 publications included, 9 showed associations of occupational sitting with cardiometabolic outcomes for the overall sample. Furthermore, the 27 publications allow to answer questions regarding subgroups. Risk estimates seem to be higher in at-risk subgroups (eg, Allesøe $e t a l^{39}$ ). Four of six publications that considered occupational sitting together with a lack of sufficient LTPA reported a higher risk of cardiometabolic outcomes for the combination of both exposures.

The informative value of this review seems to be mainly restricted by the extreme heterogeneity of both measurements and reference categories of occupational sitting as well as the variety of measured outcomes considered in the studies included. Furthermore, the spectrum of study participants' work was broad ranging from population samples to single occupations, for example, one highquality study included nurses only. ${ }^{38} 39$

Despite including longitudinal studies from the past 10 years, and the rapid literature update in PubMed, ${ }^{53-56}$ the results remained inconclusive, similar to the earlier systematic review. ${ }^{7} \mathrm{~A}$ recent comprehensive systematic review ${ }^{6}$ identified differences in physical activity, sedentary behaviour and cardiometabolic health and fitness across occupational groups (including professional drivers and office workers). Cardiometabolic outcomes investigated in studies (included in the above-mentioned review) with different designs, including cross-sectional and experimental studies, included BMI, waist-to-hip ratio, body fat percentage, blood lipids and blood glucose. ${ }^{6}$ Clinical cardiovascular outcomes (such as MI) and mortality were not considered. Prince $e t a t^{6}$ stated that the relationship between occupational sitting time and cardiometabolic health, with considerations of the effect of sex and LTPA, require investigation. The present review contributes to addressing these research needs.

The inconclusive results of occupational sitting are in contrast to the conclusive results of the relationship between cardiovascular outcomes and overall sedentary behaviour (work and leisure time) in the general population. ${ }^{57} 58$ This may be due to the difference between occupational sitting and sitting in other domains (eg, $\mathrm{TV}$ viewing). ${ }^{59}$ Moreover, the heterogeneity of exposure measurements using different concepts and questionnaires, and comparisons with different reference categories in these studies, differs from the more homogeneous exposure assessment for overall sedentary behaviour.

Underestimation of risk due to misclassification of exposure remains an issue. The reference category in four of the seven high-quality studies (eight publications) comparing different intensities of OPA was heavy or moderate manual work. Four of the seven high-quality 
studies considering different duration of sitting time also used different categories. Description of exposure was comparable in the total number of studies. While LTPA is well-documented to promote health, ${ }^{60}$ it is increasingly recognised that highly strenuous OPAs are associated with CVDs and premature mortality. ${ }^{61}{ }^{62}$ Additionally, six of the eight high-quality publications (and most of all publications included) examined the general working population, without details on occupational groups or contexts.

Thus, occupational sitting presumably also included sitting in forced postures, for example, professional driving. Occupations with highly strenuous OPAs and sitting in forced postures are related to lower SES, which is associated with cardiovascular risk factors. ${ }^{6364}$ Thus, further risk attenuation may be attributed to confounding by SES.

Nonetheless, at least half of the high-quality studies suggested an association with the primary or secondary outcome. Thus, there is a need for addressing occupational sitting to prevent cardiometabolic disease. Since none of the included studies examined device-measured occupational sitting time, quantitative recommendations for stakeholders in the field of occupational safety and health could not be deduced. To date, examples ${ }^{65-67}$ for interventions addressing occupational sitting have shown promising possibilities, based on objective measurements, and require further evaluation. Findings on sex/gender differences were inconclusive and do not support a more pronounced association in men or in women.

\section{Strengths and limitations}

This study used a comprehensive search strategy in 10 databases, with a sophisticated search string for occupational determinants of disease ${ }^{19}$ and adhered to the PRISMA guidelines. ${ }^{18}$ This review involved independent reviewers working in tandem at different stages of the review process. Moreover, it was limited to longitudinal studies and focused on sex/gender aspects of the association, contributing to an important but often neglected aspect of occupational health.

It could be disputed whether studies measuring occupational sitting as the lowest level of OPA should be included. This measurement assesses occupational and physical activity, but is not validated as a measure of sedentary behaviour in the workplace. Based on van Uffelen $e t a l^{7}$ we included studies with self-estimated duration of sitting, studies applying a job-exposure matrix and objective measurement (although none of the included studies used the latter). Considering only those studies that quantified occupational sitting time, the overall findings of associations between occupational sitting and cardiometabolic risk factors remained. Nevertheless, no definition of subgroups (eg, persons already at risk, persons with low LTPA) was a priori included in the study protocol. Moreover, because of the heterogeneity of the included studies, a meta-analysis was not reasonable.

\section{CONCLUSION}

Given the heterogeneity of exposure measurements in studies included in this review, future research should focus on using a standardised, valid and reliable measurement of occupational sitting. Combining self-reported measures with device-measured data have been recommended for improving the comprehensiveness and accuracy of sedentary behaviour measurements, ${ }^{68} 69$ and result reliability. Studies should also include a sex/gender perspective.

Subgroup analyses of at-risk individuals (eg, groups with existing health issues) might deserve further attention. Individuals with extended occupational sitting time combined with low LTPA may have particularly high risk. At least 6 hours per week of physical activity is needed to compensate for the risk imposed by sitting $>8$ hours per day. ${ }^{70}$ WHO recommends about 3 hours per week LTPA for adults. ${ }^{71}$ Doubling this amount is difficult for employees with full-time sedentary employment, particularly those with family responsibilities. Given the low adherence to the physical activity recommendations, ${ }^{72}$ preventive measures in all settings are needed.

To date, only the effect of sedentary behaviour in general, and not specifically of occupational sitting, on cardiovascular outcomes is known. Thus, interventions reducing prolonged sitting periods during both leisure and work time are recommended. From a public health perspective, the workplace is an ideal setting for health promotion and interventions, particularly for hard-toreach populations, given the amount of sitting time spent at work. ${ }^{6}$ Since this review does not exclude association of occupational sitting with cardiometabolic risk, further high-quality studies are needed, particularly as sedentary work is expected to increase in digital workplaces in future.

Acknowledgements Dr Falk Liebers, BAuA, contributed to the research proposal as an occupational health physician. Corinna Traoré supported literature management as a student research assistant.

Contributors All authors contributed to the conception of the review, analysis and interpretation of the results, and gave final approval for the manuscript. Study design: E-MB, UL and TK. Literature search and assessment (acquisition of data): KR, MP, E-MB and UL. Drafting the manuscript: KR. Critical revision of the manuscript for intellectual content: UL, E-MB, TK and MP.

Funding This work was jointly funded by the BAuA Federal Institute for Occupational Safety and Health (research project F 2399) and the German Federal Ministry of Education and Research, grant ID FKZ: 01GL1721.

Competing interests TK reports having contributed to an advisory board of CoLucid and a research project funded by Amgen, for which the Charité Universitätsmedizin Berlin received unrestricted compensation. He further reports having received honoraria from Lilly, Newsenselab and Total for providing methodological advice, from Novartis and from Daiichi Sankyo for providing a lecture on neuroepidemiology and research methods, and from the BMJ for editorial services. All other authors declare no competing interests.

Patient consent for publication Not applicable.

Ethics approval This study does not involve human participants.

Provenance and peer review Not commissioned; externally peer reviewed.

Data availability statement All data relevant to the study are included in the article or uploaded as supplementary information. 
Supplemental material This content has been supplied by the author(s). It has not been vetted by BMJ Publishing Group Limited (BMJ) and may not have been peer-reviewed. Any opinions or recommendations discussed are solely those of the author(s) and are not endorsed by BMJ. BMJ disclaims all liability and responsibility arising from any reliance placed on the content. Where the content includes any translated material, BMJ does not warrant the accuracy and reliability of the translations (including but not limited to local regulations, clinical guidelines, terminology, drug names and drug dosages), and is not responsible for any error and/or omissions arising from translation and adaptation or otherwise.

Open access This is an open access article distributed in accordance with the Creative Commons Attribution Non Commercial (CC BY-NC 4.0) license, which permits others to distribute, remix, adapt, build upon this work non-commercially, and license their derivative works on different terms, provided the original work is properly cited, appropriate credit is given, any changes made indicated, and the use is non-commercial. See: http://creativecommons.org/licenses/by-nc/4.0/.

\section{ORCID iDs}

Kathrin Reichel http://orcid.org/0000-0003-2864-9621

Tobias Kurth http://orcid.org/0000-0001-7169-2620

\section{REFERENCES}

1 WHO. Cardiovascular Diseases [Fact Sheet], 2017. Available: https:// www.who.int/en/news-room/fact-sheets/detail/cardiovasculardiseases-(cvds) [Accessed 11 Sept 2019].

2 Ekelund U, Tarp J, Steene-Johannessen J, et al. Dose-Response associations between accelerometry measured physical activity and sedentary time and all cause mortality: systematic review and harmonised meta-analysis. BMJ 2019;366:14570.

3 Biswas A, Oh PI, Faulkner GE, et al. Sedentary time and its association with risk for disease incidence, mortality, and hospitalization in adults: a systematic review and meta-analysis. Ann Intern Med 2015;162:123-32.

4 Wilmot EG, Edwardson CL, Achana FA, et al. Sedentary time in adults and the association with diabetes, cardiovascular disease and death: systematic review and meta-analysis. Diabetologia 2012;55:2895-905

5 Tremblay MS, Aubert S, Barnes JD, et al. Sedentary Behavior Research Network (SBRN) - Terminology Consensus Project process and outcome. Int J Behav Nutr Phys Act 2017;14:75.

6 Prince SA, Elliott CG, Scott K, et al. Device-measured physical activity, sedentary behaviour and cardiometabolic health and fitness across occupational groups: a systematic review and meta-analysis. Int J Behav Nutr Phys Act 2019;16:30.

7 van Uffelen JGZ, Wong J, Chau JY, et al. Occupational sitting and health risks: a systematic review. Am J Prev Med 2010;39:379-88.

8 EUGenMed Cardiovascular Clinical Study Group, Regitz-Zagrosek V Oertelt-Prigione S, et al. Gender in cardiovascular diseases: impact on clinical manifestations, management, and outcomes. Eur Heart J 2016;37:24-34

9 Hands B, Parker H. Male and female differences in health benefits derived from physical activity: implications for exercise prescription. $J$ Womens Health Issues Care 2016;5:4.

10 O'Donoghue G, Perchoux C, Mensah K, et al. A systematic review of correlates of sedentary behaviour in adults aged 18-65 years: a socio-ecological approach. BMC Public Health 2016;16:163.

11 Rhodes RE, Mark RS, Temmel CP. Adult sedentary behavior: a systematic review. Am J Prev Med 2012;42:e3-28.

12 ElfGE. Gender segregation in education, training and the labour market. Brussels: Council of the European Union, 2017: 95.

13 Jahn I, Gansefort D, Kindler-Röhrborn A. Sex-and gender-sensitive research in epidemiology and medicine: how can this be achieved? Aims and first results of the network" Sex-/Gender-Sensitive Research in Epidemiology, Neurosciences and Genetics/Cancer Research". Bundesgesundheitsblatt Gesundheitsforschung Gesundheitsschutz 2014;57:1038-46.

14 Ruiz-Cantero MT, Vives-Cases C, Artazcoz L, et al. A framework to analyse gender bias in epidemiological research. J Epidemiol Community Health 2007;61 Suppl 2:ii46-53.

15 Doull M, Welch V, Puil L, et al. Development and evaluation of 'briefing notes' as a novel knowledge translation tool to aid the implementation of sex/gender analysis in systematic reviews: a pilot study. PLoS One 2014;9:e110786.

16 Artazcoz L, Borrell C, Cortès I, et al. Occupational epidemiology and work related inequalities in health: a gender perspective for two complementary approaches to work and health research. $J$ Epidemiol Community Health 2007;61 Suppl 2:ii39-45.
17 Reichel K, Latza U, Backé EM. The impact of occupational sitting on cardiometabolic risk factors and cardiometabolic outcomes-A systematic review from a gender-sensitive perspective.: prospero, 2018. Available: http://www.crd.york.ac.uk/PROSPERO/display record.php?ID = CRD42018079219 [Accessed 11 Sept 2019]

18 Moher D, Liberati A, Tetzlaff J. Preferred reporting items for systematic reviews and meta-analyses: the PRISMA statement. Ann Intern Med 2009;151:264.

19 Mattioli S, Zanardi F, Baldasseroni A, et al. Search strings for the study of putative occupational determinants of disease. Occup Environ Med 2010;67:436-43.

20 Pulsford RM, Stamatakis E, Britton AR, et al. Sitting behavior and obesity: evidence from the Whitehall II study. Am J Prev Med 2013;44:132-8.

21 Pulsford RM, Stamatakis E, Britton AR, et al. Associations of sitting behaviours with all-cause mortality over a 16-year follow-up: the Whitehall II study. Int J Epidemiol 2015;44:1909-16.

22 Stamatakis E, Pulsford RM, Brunner EJ, et al. Sitting behaviour is not associated with incident diabetes over 13 years: the Whitehall II cohort study. Br J Sports Med 2017;51:818-23.

23 van der Ploeg HP, Møller SV, Hannerz H, et al. Temporal changes in occupational sitting time in the Danish workforce and associations with all-cause mortality: results from the Danish work environment cohort study. Int J Behav Nutr Phys Act 2015;12:71.

24 Eriksen D, Rosthøj S, Burr H, et al. Sedentary work--associations between five-year changes in occupational sitting time and body mass index. Prev Med 2015;73:1-5.

25 Møller SV, Hannerz H, Hansen AM, et al. Multi-wave cohort study of sedentary work and risk of ischemic heart disease. Scand $J$ Work Environ Health 2016;42:43-51.

26 Held C, Iqbal R, Lear SA, et al. Physical activity levels, ownership of goods promoting sedentary behaviour and risk of myocardial infarction: results of the INTERHEART study. Eur Heart $J$ 2012;33:452-66.

27 Cheng X, Li W, Guo J, et al. Physical activity levels, sport activities, and risk of acute myocardial infarction: results of the INTERHEART study in China. Angiology 2014;65:113-21.

28 European Union. EU languages, 2020. Available: https://europa. eu/european-union/about-eu/eu-languages_en [Accessed 18 Nov 2020].

29 SIGN. Critical appraisal notes and checklists, 2014. Available: https:// www.sign.ac.uk/what-we-do/methodology/checklists/ [Accessed 04 Apr 2019].

30 Kumar A, Prasad M, Kathuria P. Sitting occupations are an independent risk factor for ischemic stroke in North Indian population. Int J Neurosci 2014;124:748-54.

31 Selim S, Rahman R, Yasmin R, et al. Risk factors of acute coronary syndrome among Bangladeshi people. Mymensingh Med $\mathrm{J}$ 2013;22:513-21.

32 Martin KR, Kuh D, Harris TB, et al. Body mass index, occupational activity, and leisure-time physical activity: an exploration of risk factors and modifiers for knee osteoarthritis in the 1946 British birth cohort. BMC Musculoskelet Disord 2013;14:219.

33 Thompson WG, St Sauver J, Schroeder D. Occupation, sitting, and weight change in a cohort of women employees. J Occup Environ Med 2018;60:44-7

34 Kim Y, Wilkens LR, Park S-Y, et al. Association between various sedentary behaviours and all-cause, cardiovascular disease and cancer mortality: the Multiethnic cohort study. Int J Epidemiol 2013;42:1040-56.

35 Chau JY, Grunseit A, Midthjell K, et al. Sedentary behaviour and risk of mortality from all-causes and cardiometabolic diseases in adults: evidence from the HUNT3 population cohort. Br J Sports Med 2015;49:737-42.

36 Hayashi R, Iso H, Cui R, et al. Occupational physical activity in relation to risk of cardiovascular mortality: the Japan collaborative cohort study for evaluation for cancer risk (JACC study). Prev Med 2016;89:286-91.

37 Smith P, Ma H, Glazier R. The relationship between prolonged occupational standing and sitting and incident cardiovascular disease. Eur J Prev Cardiol 2017;24:21.

38 Allesøe K, Holtermann A, Aadahl M, et al. High occupational physical activity and risk of ischaemic heart disease in women: the interplay with physical activity during leisure time. Eur J Prev Cardiol 2015;22:1601-8.

39 Allesøe K, Søgaard K, Aadahl M, et al. Are hypertensive women at additional risk of ischaemic heart disease from physically demanding work? Eur J Prev Cardiol 2016;23:1054-61.

40 Kikuchi $\mathrm{H}$, Inoue S, Odagiri Y, et al. Occupational sitting time and risk of all-cause mortality among Japanese workers. Scand J Work Environ Health 2015;41:519-28. 
41 Saidj M, Jørgensen T, Jacobsen RK, et al. Work and leisure time sitting and inactivity: effects on cardiorespiratory and metabolic health. Eur J Prev Cardiol 2016;23:1321-9.

42 Hall C, Heck JE, Sandler DP, et al. Occupational and leisure-time physical activity differentially predict 6-year incidence of stroke and transient ischemic attack in women. Scand $J$ Work Environ Health 2019;45:267-79.

43 Sakaue A, Adachi H, Enomoto M, et al. Association between physical activity, occupational sitting time and mortality in a general population: an 18-year prospective survey in Tanushimaru, Japan. Eur J Prev Cardiol 2020;27:758-766.

44 Saltin B, Grimby G. Physiological analysis of middle-aged and old former athletes. Comparison with still active athletes of the same ages. Circulation 1968;38:1104-15.

45 Moe B, Mork PJ, Holtermann A, et al. Occupational physical activity, metabolic syndrome and risk of death from all causes and cardiovascular disease in the HUNT 2 cohort study. Occup Environ Med 2013;70:86-90.

46 Stamatakis E, Chau JY, Pedisic Z, et al. Are sitting occupations associated with increased all-cause, cancer, and cardiovascular disease mortality risk? A pooled analysis of seven British population cohorts. PLoS One 2013;8:e73753.

47 Ferrario MM, Roncaioli M, Veronesi G, et al. Differing associations for sport versus occupational physical activity and cardiovascular risk. Heart 2018;104:1165-72.

48 Johnsen AM, Alfredsson L, Knutsson A, et al. Association between occupational physical activity and myocardial infarction: a prospective cohort study. BMJ Open 2016;6:e012692.

49 Ma Y, Wang Y-J, Chen B-R, et al. Study on association of working hours and occupational physical activity with the occurrence of coronary heart disease in a Chinese population. PLoS One 2017;12:e0185598.

50 Lin T-chi, Courtney TK, Lombardi DA, et al. Association between sedentary work and BMI in a U.S. national longitudinal survey. Am J Prev Med 2015;49:e117-23.

51 Picavet HSJ, Pas LW, van Oostrom SH, et al. The Relation between Occupational Sitting and Mental, Cardiometabolic, and Musculoskeletal Health over a Period of 15 Years--The Doetinchem Cohort Study. PLoS One 2016;11:e0146639.

52 Pinto Pereira SM, Power C. Sedentary behaviours in mid-adulthood and subsequent body mass index. PLoS One 2013;8:e65791.

53 Hermansen R, Jacobsen BK, Løchen M-L, et al. Leisure time and occupational physical activity, resting heart rate and mortality in the Arctic region of Norway: the Finnmark study. Eur J Prev Cardiol 2019;26:1636-44.

54 Hupin D, Raffin J, Barth N, et al. Even a previous light-Active physical activity at work still reduces late myocardial infarction and stroke in retired adults aged $>65$ years by $32 \%$ : The PROOF Cohort Study. Front Public Health 2019;7:51.

55 Mikkola TM, von Bonsdorff MB, Salonen MK, et al. Physical heaviness of work and sitting at work as predictors of mortality: a 26-year follow-up of the Helsinki birth cohort study. BMJ Open 2019;9:e026280.

56 Piirtola M, Kaprio J, Svedberg P, et al. Associations of sitting time with leisure-time physical inactivity, education, and body mass index change. Scand J Med Sci Sports 2020;30:322-31.
57 Patterson R, McNamara E, Tainio M, et al. Sedentary behaviour and risk of all-cause, cardiovascular and cancer mortality, and incident type 2 diabetes: a systematic review and dose response metaanalysis. Eur J Epidemiol 2018;33:811-29.

58 Ekelund U, Brown WJ, Steene-Johannessen J, et al. Do the associations of sedentary behaviour with cardiovascular disease mortality and cancer mortality differ by physical activity level? A systematic review and harmonised meta-analysis of data from 850 060 participants. Br J Sports Med 2019;53:886-94.

59 Wennman H, Vasankari T, Borodulin K. Where to sit? type of sitting matters for the Framingham cardiovascular risk score. AIMS Public Health 2016;3:577-91.

60 Leitzmann MF, Park Y, Blair A, et al. Physical activity recommendations and decreased risk of mortality. Arch Intern Med 2007;167:2453-60.

61 Holtermann A, Krause N, van der Beek AJ, et al. The physical activity paradox: six reasons why occupational physical activity (opa) does not confer the cardiovascular health benefits that leisure time physical activity does. Br J Sports Med 2018;52:149-50.

62 Holtermann A, Burr H, Hansen JV, et al. Occupational physical activity and mortality among Danish workers. Int Arch Occup Environ Health 2012;85:305-10.

63 Kaplan GA, Keil JE. Socioeconomic factors and cardiovascular disease: a review of the literature. Circulation 1993;88:1973-98.

64 Marmot MG, Smith GD, Stansfeld S, et al. Health inequalities among British civil servants: the Whitehall II study. Lancet 1991;337:1387-93.

65 Healy GN, Goode A, Schultz D, et al. The BeUpstanding Program ${ }^{\mathrm{TM}}$ : Scaling up the Stand Up Australia Workplace Intervention for Translation into Practice. AIMS Public Health 2016;3:341-7.

66 Danquah IH, Kloster S, Holtermann A, et al. Take a Stand!-a multicomponent intervention aimed at reducing sitting time among office workers-a cluster randomized trial. Int J Epidemiol 2017;46:128-40.

67 Maylor BD, Edwardson CL, Zakrzewski-Fruer JK, et al. Efficacy of a multicomponent intervention to reduce workplace sitting time in office workers: a cluster randomized controlled trial. J Occup Environ Med 2018;60:787-95.

68 Healy GN, Clark BK, Winkler EAH, et al. Measurement of adults' sedentary time in population-based studies. Am J Prev Med 2011;41:216-27.

69 Matthews CE, Moore SC, George SM, et al. Improving self-reports of active and sedentary behaviors in large epidemiologic studies. Exerc Sport Sci Rev 2012;40:118-26.

70 Ekelund U, Steene-Johannessen J, Brown WJ, et al. Does physical activity attenuate, or even eliminate, the detrimental association of sitting time with mortality? A harmonised metaanalysis of data from more than 1 million men and women. Lancet 2016;388:1302-10.

71 WHO. Global recommendations on physical activity for health [Brochure], 2010. Available: https://www.who.int/publications/i/item/ 9789241599979 [Accessed 16 Nov 2020].

72 Krug S, Jordan S, Mensink GB. Physical activity: results of the German health interview and examination survey for adults (DEGS1). Bundesgesundheitsblatt Gesundheitsforschung Gesundheitsschutz 2013;56:765-71. 